\title{
Multipath Discovery Algorithms for VoD Streaming In Wireless Mesh Network
}

\author{
${ }^{1}$ Praful C Ramteke, ${ }^{2}$ V.S.Jadhav and ${ }^{3}$ Raju Wadekar \\ ${ }^{1 \& 2}$ Maharashtra Institute of Technology, Pune, India \\ ${ }^{3}$ University of Lancaster, United Kingdom \\ ${ }^{1}$ praful.c.ramteke@gmail.com, 2vinod_jadhav@yahoo.com, ${ }^{3}$ govindraoraju@yahoo.com
}

\begin{abstract}
Transmission and routing of video data over wireless network is a challenging task because of wireless interferences. To improve the performance of video on demand transmission over wireless networks multipath algorithms are used. IPD/S (Iterative path discovery/selection) PPD/S (Parallel Path discovery/selection) are two algorithms which is used for discovering maximum number of edge disjoint paths from source to destination, for each VoD request by considering the effects of wireless interferences. In this paper performance evaluation of these multipath discovery algorithms for VoD (Video on demand) streaming in wireless mesh network is presented. These algorithms are evaluated on the bases of Number of Path discovers, Packet drop ratio and delay. Simulation result shows that PPD/S works batter as compared to IPD/S because it's able to discover more paths than IPD/S under same circumstances.
\end{abstract}

Keywords-Multisource video streaming, IPD, PPD wireless mesh network and multipath routing

\section{Introduction}

Video streaming is gaining popularity among mobile users recently. It has been forecasted that mobile video data traffic will account for $70 \%$ of total mobile data traffic by 2016[1]. One of the most used application in internet services recently is video on demand application (VoD). In this VoD application a video is provided by a respected server to the demanding users. Most of the VoD applications use peer to peer technology to increase the VoD performance. Considering video which have been watched recently by users is store in their local storage. In peer to peer technology when a user wants to watch a new video, she or he primarily discovers which peer user have buffered the video and then streams the video from both peer client and servers through multi-path. A multi-path multi-source video on demand streaming has achieve a great success in wired network but somehow remains a challenging in case of wireless network due to several wireless interferences.

As wireless networking technology is attracting more interest in research industry and building community networks due to low cost infrastructure. A community network is composed of many mesh routers where each mesh router established connectivity with its neighboring router. When a user request for a video it can stream the video from two sources 1) the server or peer that have buffered the video. 2) From the other users who have access to the required video.

Video streaming is a challenging task due to high bit rate, delay and loss sensitivity. However it is assume that by streaming video from multiple paths we can improve the performance of video streaming in wireless network. There are several peer-to-peer architecture has been proposed. [2][3][4]. Path selection is depend on two factors i.e. Path disjointness and Hop count 


\subsection{Path Disjointness}

There are several path discovering algorithms and they are discovering several paths sharing common nodes or even links. Such set of paths are less reliable because transmission failure in any of shared nodes causes loss of data streams most probably packet loss. One of the major problems in multi-source VoD applications is discovery of multiple independent path and path selection form the selected paths. The independent paths are defined as the path which have are not depending on other paths i.e. edge-disjoint or vertex-disjoint paths. In edge disjoint paths no two paths with share a same link and therefore the possibility of link failure is minimized. Vertex disjoint is stronger than edge disjointness, because it provide guarantee that node failure will affect mostly one path. In wireless mesh network it's very difficult to find independent paths due to the wireless interference. Another disadvantage of shared nodes is reduction in paths capacity, since the they aggregate traffic from several paths. This may cause self interference. Hop count has impact on two quality metricend to end delay and path reliability as the number of hop increases it increases the end to end delay. [7]

In this paper evaluation of two multipath discovery algorithms is presented. One of the major problems in multi-source VoD applications is discovery of multiple independent path and path selection form the selected paths. The independent paths are defined as the path which have are not depending on other paths i.e. edge-disjoint or vertex-disjoint paths. In edge disjoint paths no two paths with share a same link and therefore the possibility of link failure is minimized. Vertex disjoint is stronger than edge disjointness, because it provide guarantee that node failure will affect mostly one path. In wireless mesh network it's very difficult to find independent paths due to the wireless interference. All we know about wireless mesh network is that this network is designed to share among multiple users so it provides advantage while using VoD application in this network.

\section{Multisource Video on Demand in WMN}

In multi-source video on demand streaming a single required video is stream from more than one source in general in conventional video streaming video is only available through server in this case of multi source video on demand streaming video the required video is not only provided by the respected server but also by the other means of source also (from fig.1) i.e. other users. For some popular videos the VoD performance can be improved by P2P technology. In this technology whenever the user request for a video it first registers with server so that server keeps the list of visited users. In future when new user request for the same video server can provide list of previous users so that new user can not only get a video stream from the server but also from the previous users of the video.

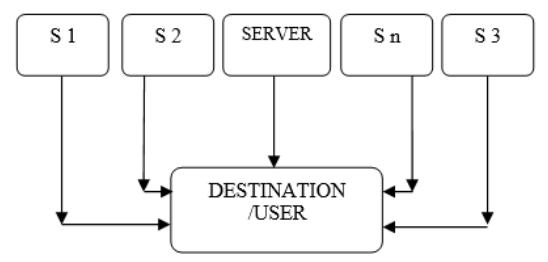

Figure 1: Multi-source VoD in wireless mesh Network

\section{Multi Path Discovery Algorithms}

In wireless mesh networks, the mobility of mesh router is minimal. For example in community networks most of the time routers are fixed on roofs of houses in addition to it due to overhead of dynamic channel switching a static channel allocation strategies are used in which channel are often 
changed. This make possible for each router to collect the global information about its network including channel assignment and position of each router.

There are several algorithms present which is used for multipath discovery in this paper we are dealing with $\mathrm{IPD} / \mathrm{S}$ and $\mathrm{PPD} / \mathrm{S}$ i.e. iterative path discovery/selection and parallel path discovery/selection algorithms.

\subsection{Iterative Path Discovery/Selection (IPD/S)}

The Iterative Path Discovery algorithm (IPD/S) finds paths one by one from the senders to the receiver. In each iteration, one path from a sender to the receiver, and then update the topology accordingly. This process continues until no new paths can be found from the remaining topology.

In Iterative path discovery algorithm paths from all source to single destination is discovered in a step by step manner. (Form fig.2)When a destination or user demand for the video then the request is first send to the respected server from the server a user collect the information about other source that are able to provide the desired video to the destination. After gathering all the information about all the available sources the Iterative path discovery starts working. Here IPD/S select a single source and then find the path towards destination after reaching at destination it updated the topology and again starts the search this time by considering other source and the search is going on till the last available source. Each time after discovering the path it is necessary to update the topology which guarantees the edge disjointedness of path.

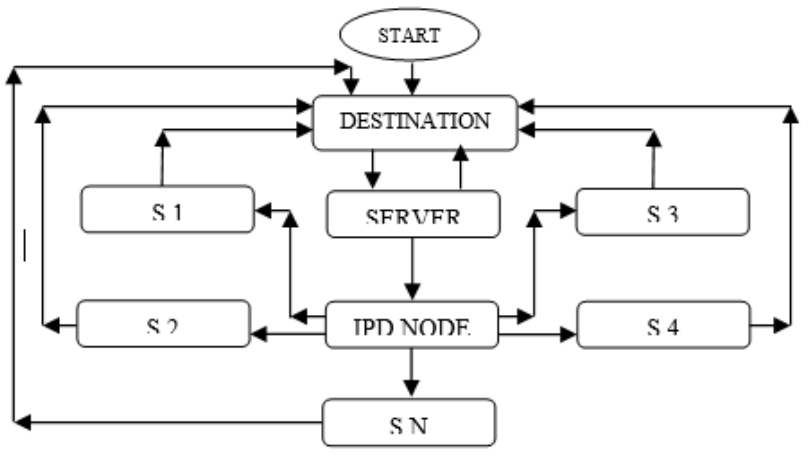

Figure 2: Block Diagram of IPD/S

\subsubsection{Path Selection}

Selection of path is done on the basis of minimum total interference, so that the selected path will render fewer changes in the remaining topology and thus leaves more flexibility for finding more paths afterwards.

Let $S$ be the set of senders and $T$ be the initial topology. Let $S^{\prime}$ be the set of remaining senders, for which no paths found yet, and $T^{\prime}$ be the remaining topology. Initially, $S^{\prime}=S$ and $T^{\prime}=T$. In this step, for each $s \in S^{\prime}$, First find a minimum WCETT path $p$ from $s$ to $r$ in $T^{\prime}$ if such a path exists and $W C E T T(p) \leq \gamma \cdot w T(s, r)$, where $w T(s, r)$ is the WCETT value of the optimal path from $s$ to $r$ in $T$ and $\gamma$ is a constant to control the quality of each path. Denote the resulting set of paths as $P$, after gating the resulting set of paths next step is to decide which path to select from set of paths $P$. The total interference of $p$ in network is denoted by $I F T^{\prime}(p)$, the interfere with any edge in $p$ with respect to the number of edges in $T^{\prime}$ is given as

$$
\begin{gathered}
I F T^{\prime}(p)=\mid\left\{e^{\prime} \mid e^{\prime} \in E^{\prime} \wedge \exists e \in p: \text { Interfere }\left(e, e^{\prime}\right)\right\} \mid \\
\left(e, e^{\prime}\right) \text { is true if } e=e^{\prime}
\end{gathered}
$$


In VoD applications, it's not only about finding more disjoint paths from source to destination but also need to guarantee the quality of each path with respect to packet loss, delay and throughput. There are several metric present for finding good routes between single source to destination in wireless network for example The WCETT metric [9] is widely used in multichannel multiinterface wireless mesh networks. It not only considers packet loss and delay, but also accounts for channel diversity in each path so as to reduce intraflow interference. Here we are using this metric to evaluate the quality of each selected path it not only considers packet loss and delay, but also accounts for channel diversity in each path so as to reduce intraflow interference. Therefore, by using this metric to evaluate the quality of each selected path. while keeping the WCETT value of each path below a certain threshold. The WCETT metric of a path can be calculated as follows:

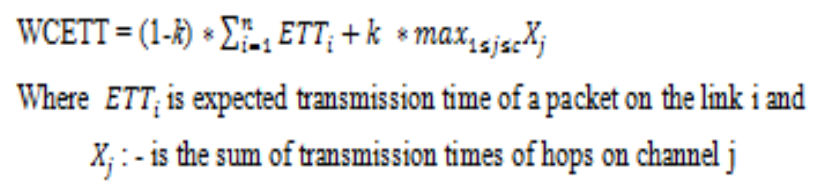

\subsubsection{Topology Update}

Once a path has been selected, it needs to update accordingly. Updating the topology guarantees the edge disjointedness of paths, so that the paths found later will not overlap with the paths previously found. In addition to this it needs to consider interference on topology and guarantee the level of independency among the final set of selected paths.

Consider a label $l$ on the edges of $T^{\prime}$ to record the interference from the already selected paths, where $(e)$ counts how many selected paths are interfering with link $e$. At the start of the algorithm, $(e)$ is initialized to 0 . Assume $p$ is the selected path from $T^{\prime}\left(V, E^{\prime}\right)$ in the current iteration. We define the path interfering set of $p$ in $T^{\prime}$, denoted by $I E T^{\prime}(p)$, as the set of edges in $\left(T^{\prime}-p\right)$ that interfere with any edge in $p$.

$$
\operatorname{IET^{\prime }}(p)=\left\{e^{\prime} \mid e^{\prime} \in\left(E^{\prime}-p\right) \wedge \exists e \in p: \text { Interfere }\left(e, e^{\prime}\right)\right\}
$$

By updating $(e)$ for each edge $e \in I E T^{\prime}(p)$ by increasing 1 , indicating that there is one more selected path $p$ that interferes with $e$. If $l(e)>\alpha$, then by taking off $e$ from $T^{\prime}$.This is because if $e$ has been used in one more path in the remaining topology, then $e$ will interfere with more than $\alpha$ already selected paths, which violates the constraint in the COMINP problem.

Constrained Maximum Independent Paths problem (COMINP) the maximum number of edgedisjoint paths from $S$ to $D$, such that

1. $\mathrm{MPI}(\mathrm{P}) \leq \alpha$ where $\alpha$ is the threshold to control the level of independency between path in $P$

2. $\operatorname{WCETT}_{(P)} \leq \mu$ for $\mathrm{p} \in \mathrm{P}$, where $\mu$ is the threshold to control the quality of each path

\subsubsection{Parallel Path Discovery/ Selection (PPD/S)}

In parallel path discovery algorithm paths from all source to single destination is discovered in a single step. 


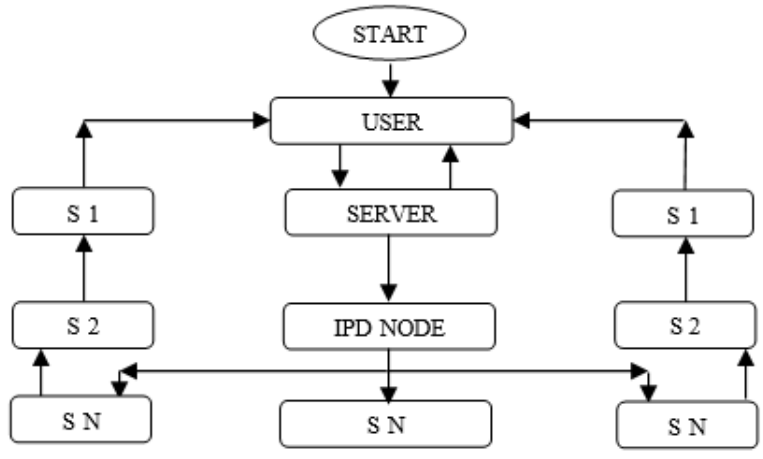

Figure 3: Block Diagram of PPD/S

Here as shown in Figure 3 when a destination or user demand for the video then the request is first send to the respected server from the server a user collect the information about other source that are able to provide the desired video to the destination.

After gathering all the information about all the available sources the Parallel path discovery starts working. PPD collects information about entire available source for the video transfer from the server and starts discovering the paths from each source to a destination. This is totally different from the IPD as in this algorithm all the paths are get discovered in a single step by this the time required to perform other operation is reduced. At the end of algorithm the information is get updated in a PPD node and the most appropriate path is get selected for the video transfer, while selecting the path the most important thing to consider is the paths need to be edge disjoint (i.e. the paths need to be independent of each other). In the Internet, the independent paths are usually defined as edge-disjoint or vertex-disjoint paths. In edge-disjoint paths, no two paths share a same link, and therefore any link failure will only affect one path.

\section{Simulation Methodology}

Simulation is performed by using NS2. To support multiple interfaces and multiple channels per node in simulation Hyacinth extension [5] has been used. During network transmission the frames are encapsulated into User Defined Protocol (UDP) packets and reconstructed at the receiver. In all the simulation 802.11 MAC protocol is been used with CBR (Constant Bit Rate) and UDP with the maximum packet size of 512 bit for multimedia data transport. A simulation is performed in 20, 40 and 60 nodes random topology within an area $500 \times 500$.There are four channels used in channel assignment. The channel allocation algorithm proposed in [6] is used to statistically assign channels for each interference in order to minimize the wireless interference within the network.

There are dependencies between the encoded video frames for ex. Any group of picture (GOP) is made up of three frames I, B and P. I-frames in GoP is required to decode all other frames $P$ and $B$ in a GoP and $P$-frames are required to decode all successive $P$ as well as $B$-frames encoded with respect to these P-frames. So even if a frame has been received before deadline it is regarded as not constructed if its dependent frames are not reconstructed successfully.

Here we compare the following method of path discovery for multi-source video on demand streaming.

1. IPD/S: Used the Iterative path Discovery/selection algorithm to find the maximum number of edge disjoint independent path.

2. PPD/S: Used the Parallel Path discovery/Selection algorithm to find the maximum number of edge disjoint path. 


\section{Performance Evalutation}

In this section result Based on NS2 simulation has been presented.

\subsection{Number of Paths}

For discovering number of paths in a network a single router is selected as a receiver in network and randomly designed $\mathrm{n}$ routers are selected as senders. While discovering a path by IPD/S and PPD/S edge-disjoint paths are only considered at the same time while selecting edge disjoint paths algorithms are performed for $\beta=0$ and $\beta=1$ i.e. at $\beta=0$ no path interfere with each other whereas at $\beta=1$ each link of any path interferes at most with one other path among the multiple path finally discover. The number of paths discovered by IPD/S and PPD/S under different number of senders are shown in figure [4] and [5].As we can observe PPD/S is able to discover more paths than IPD/S under the same constraints.

\subsection{Packet drop Ratio}

When a user streams video over multiple paths, it requires to determine the playout deadline that is the time the user waits before playing the video. There are two main reasons due to which packet drop occurs in a network. 1) When packet is loss due to interference in wireless transmission or collision. 2) The packet is received successfully but it's received after deadline time.

\subsection{Delay jitter}

Network delay is an important design and performance characteristic of a computer network or a telecommunication network. The delay of a network specifies how long it takes for a bit of data to travel across the network from one node to another or end point and if the network reaches its maximum capacity further $\mathrm{VoD}$ request are blocked [8]

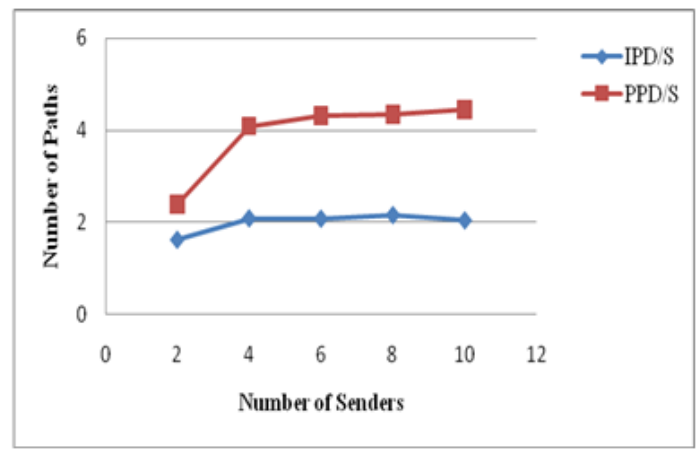

Figure 4: The Number of Paths Discovered $(\beta=0)$

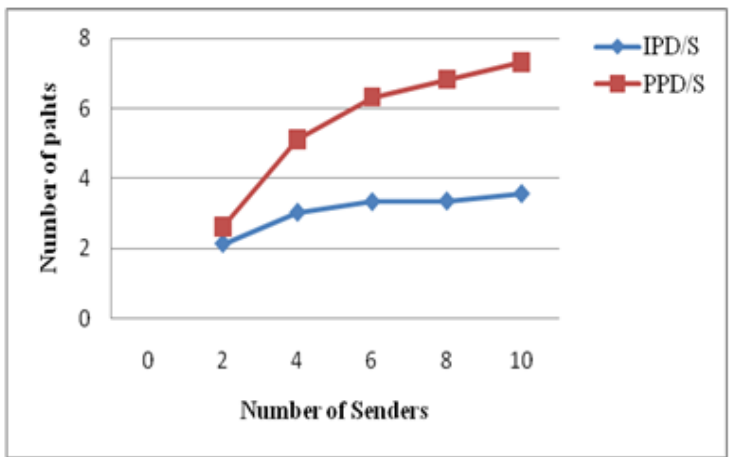

Figure 5: The Number of Paths Discovered $(\beta=1)$

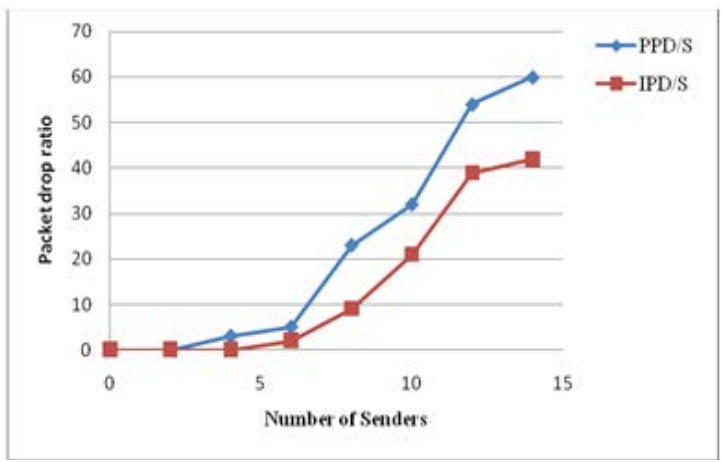

Figure 6: Packet drop ratio (payout deadline=10ms) 


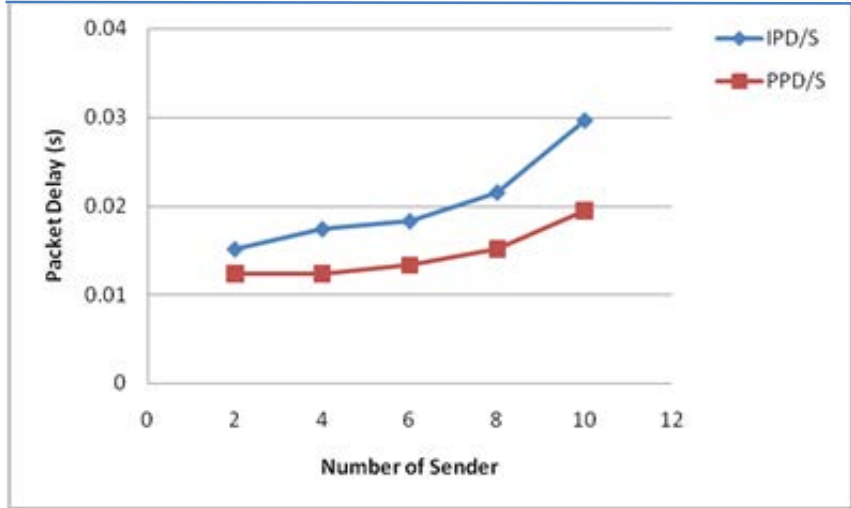

Figure 7: Packet Delay over number of senders

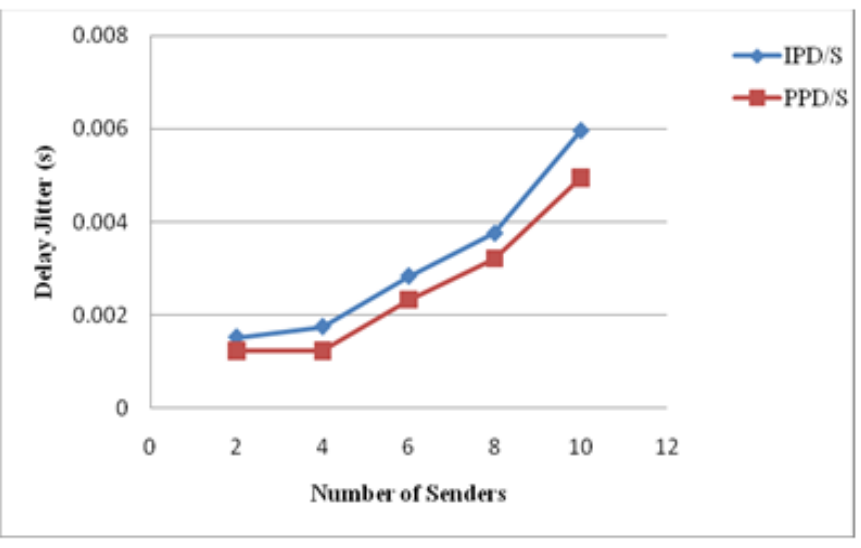

Figure 8: Delay Jitter over number of senders

\section{Conclusion}

In this paper two multipath discovery algorithms, IPD/S and PPD/S to find multiple independent paths from sender to receiver for each VoD request are studied. The simulations are performed on NS2 and evaluate the performance of algorithms. Simulation result has shown that PPD/S achieves better video streaming performance than IPD/S, because it is able to discover more paths than IPD/S under same conditions.

\section{REFERENCES}

[1]. Cisco, Cisco visual networking index: Global mobile data traffic forecast update, 2011-2016.

[2]. D. A. Tran, K. A. Hua, and T. T. Do, "A peer-to-peer architecture for media streaming," IEEE J. Sel. Areas Commun., vol. 22, no. 1, pp.121-133, Jan. 2004.

[3]. T. Nguyen and A. Zakhor, "Multiple sender distributed video streaming," IEEE Trans. Multimedia, vol. 6, no. 2, pp. 315-326, Apr.2004.

[4]. Y. Zhu, W. Zeng, H. Liu, Y. Guo, and S. Mathur, "Supporting video streaming services in infrastructure wireless mesh networks: Architecture and protocols," in Proc. IEEE ICC, 2008, pp. 1850-1855.

[5]. T.-C. Chiueh, A. Raniwala, R. Krishnan, and K. Gopalan, "Hyacinth: An IEEE 802.11-based multi-channel wireless meshnetwork," 2005 [Online] .Available: http://www.ecsl.cs.sunysb.edu/multichannel

[6]. A. P. Subramaniam, H. Gupta, and S. R. Das, "Minimum-interference channel assignment in multi-radio wireless mesh networks," in Proc.IEEE SECON, 2007, pp. 481-490.

[7]. Lauris Cikovski and IImars Slaidins."Analysis of wireless Ad-hoc Network Parameters for Efficient Multipath Video transfer." In IEEE 2012

[8]. X. Zhu, S. Han, and B. Girod, "Congestion-aware rate allocation for multipath video streaming over ad hoc wireless networks," in Proc IEEE ICIP, 2004, vol. 4, pp. 2547-2550 
[9]. R. Draves, J. Padhye, and B. Zill, "Routing in multi-radio multi-hop wireless mesh networks," Proc. ACM MobiCom, 2004, pp. 114-128.

[10]. J. Tang, G. Xue, and W. Zhang, "Interference-aware topology control and QoS routing in multi-channel wireless mesh networks," in Proc.ACM MobiHoc, 2005, pp. 68-77.

[11]. Y. Ding, Y. Yang, and L. Xiao, "Multi-path routing and rate allocation for multi-source video on-demand streaming in wireless mesh networks," in Proc. IEEE INFOCOM, 2011, pp. 20512059.

[12]. S. J. Lee and M. Gerla, "Split multipath routing with maximally disjoint paths in ad hoc networks," in Proc. IEEE ICC, 2001, vol. 10, pp.3201-3205.

[13]. M. Marina and S. Das, "On-demand multipath distance vector routing in ad hoc networks," in Proc. IEEE ICNP, 2001, pp. 14-23.

[14]. D. B. Johnson and D. A. Maltz, "Dynamic source routing in ad hoc wireless networks," in Proc. Mobile Comput., 1996, pp. 153-181. 